\section{THU0427 AMBIENT AIR POLLUTION AND RISK OF ACUTE GOUT FLARES; A TIME-SERIES STUDY}

H.J. Ryu ${ }^{1}$, M.R. Seo ${ }^{1}$, H.J. Choi ${ }^{1}$, J. Cho ${ }^{2}$, H.J. Baek ${ }^{1} .{ }^{1}$ Rheumatology; ${ }^{2}$ Occupational and Environmental Medicine, Gachon University Gil Medical Center, Incheon, Korea, Republic Of

Background: Air pollution is caused by substances that consists of solid particles, liquid droplets, or gases including carbon monoxide (CO), nitrate (NO), sulfur dioxide $\left(\mathrm{SO}_{2}\right)$, ozone $\left(\mathrm{O}_{3}\right)$, lead, toxic product from tobacco smoke and particulate matter (PM). Inhaled air pollutants can induce oxidative stress and this can contribute to trigger or exacerbate systemic inflammation and autoimmunity. The relations of air pollution with rheumatic diseases have been reported, especially in rheumatoid arthritis, systemic lupus erythematosus, systemic sclerosis and juvenile idiopathic arthritis. Gout is an inflammatory disease caused by deposition of monosodium urate (MSU) crystals. Acute gout flares is initiated with activation of the NLRP 3 inflammasome and release of IL-1 $\beta$ by MSU crystals. PM $<10 \mu \mathrm{m}$ in diameter $\left(\mathrm{PM}_{10}\right)$ exposure has been reported to activate NLRP3 inflammasome in airway epithelial cells. The influence of air pollutants including $\mathrm{PM}_{10}$ to acute gout flares has not yet been known.

Objectives: To investigate the association between air pollution and acute gout flares

Methods: We obtained data from the National Health Insurance Database between 2007 and 2015 in Incheon, Republic of Korea by Health Insurance Review and Assessment (HIRA) service. The HIRA data included age, gender, national health insurance type, diagnosis code based on the International Classification of Diseases version 10 (ICD-10), visit date, procedure code, and prescription code. We studied the subjects of age $>19$ years who visited emergency department (ED) due to acute gout flare between 2008 and 2015. Acute gout flare was defined as an ED visit due to gout (ICD-10, M10) with any prescription of non-steroidal anti-inflammatory drugs (NSAIDs), acetaminophen, steroids, or colchicine. Comorbidities and prescription history were collected. The data of ambient $\mathrm{PM}_{10}, \mathrm{O}_{3}, \mathrm{NO}_{2}, \mathrm{CO}$, and $\mathrm{SO}_{2}$ levels were obtained from the Ministry of Environment data sources. We performed time-series study. The generalized additive models with Poisson distribution were used to investigate the association between single air pollutant level and acute gout flares. To examine the delayed effect of air pollutant exposure before the ED visit day on acute gout flare, we established cumulative lag models (averaging levels of consecutive days).

Results: The total number of ED visits for acute gout flare from 2008 to 2015 in Incheon was 139,665 , including $48.8 \%$ of $40-59$ year-old men. The ED visits for acute gout attack were most likely to occur in summer (29.1\%). Among the ED cases of acute gout flares, $11.0 \%$ had a prescription history of allopurinol or febuxostat for the six months before the visit. The relative risks (RRs) of $\mathrm{PM}_{10}$ and $\mathrm{O}_{3}$ at lag0-7 were $1.02(1.01-1.03)$ and $1.07(1.06-1.08)$, respectively. $\mathrm{NO}_{2}, \mathrm{CO}$, and $\mathrm{SO}_{2}$ did not show significant association with acute gout flares. Acute gout flares showed more significant association with $\mathrm{O}_{3}$ in the cases with hypertension and use of NSAIDs, colchicine, or statins. $\mathrm{PM}_{10}$ was more significantly associated with acute gout flares in the cases with congestive heart failure.

Conclusions: Ambient air pollution can induce acute gout flares and its influence on acute gout flares may increase according to the comorbidities or medications. Disclosure of Interest: None declared

DOI: 10.1136/annrheumdis-2017-eular.6188

\section{THU0428 ON THE INCIDENCE OF GOUT FLARES IN PRIMARY CARE: A RETROSPECTIVE COHORT STUDY}

$\underline{\text { H. Janssens }}{ }^{1}$, M. Janssen ${ }^{2}$, J. Fransen ${ }^{3}$, M. Flendrie ${ }^{4} .{ }^{1}$ Department of Primary and Community Care, Radboud University Medical Centre, Nijmegen; ${ }^{2}$ Rheumatology, Rijnstate Hospital, Arnhem; ${ }^{3}$ Radboud University Medical Centre; ${ }^{4}$ Rheumatology, Sint Maartenskliniek, Nijmegen, Netherlands

Background: From rheumatologist point of view (secondary care) there is a serious concern about suboptimal treatment of gout patients in primary care with warnings for worse prognostic consequences. ${ }^{(1)}$ Some refer even to a "state of suboptimal gout care" promoted by "substantial gaps between rheumatologists and primary care providers" in their approaches to gout care:(2) the strategy of "treating-to-target" (to lower serum uric acid (SUA) levels $<0.36 \mathrm{mmol} / \mathrm{L}$ ) versus "treating-to-avoid-symptoms" (often without addressing hyperuricemia). (3) Most studies on gout care in the primary care setting checked patients for prescribed urate lowering treatment (ULT), SUA assessments or reached SUA target levels, omitting the major patient related clinical end point of gout, flare frequency. ${ }^{(4)}$ Objectives: To quantify the occurrence of flares and the use of ULT, in primary care patients with gout, and to analyze patient characteristics related to low or high flare frequency.

Methods: A retrospective cohort study (setting one Dutch primary care center with an integrated medical praxis and pharmacy). Electronic medical records of ca. 5800 enlisted patients were used to select all patients with gout, to analyze their ULT use, and to assess flare frequency in a 2-year time window (2014-2015). Flare was defined as each pharmacy delivery of an anti-inflammatory drug or pain killer linked to a morbidity code for gout. Associations were studied between high or low flare frequency and patient characteristics by univariate logistic regression. Results: Of 173 included patients (prevalence 3\%; mean age $66.4 \mathrm{yr} ; 75.7 \%$ men) $38.7 \%$ used ULT persistently during the 2 -year time-window. Median time after initial diagnosis was $8.0 \mathrm{yr}$ (IQR 3.0-14.5). Mean total numbers of flares in two years was 2.7 (SD: 4.7), median 1.0 (IQR: 0.0-4.0). Of the patients not receiving ULT $(n=106,61.3 \%) 41.5 \%$ had never, $25.5 \%$ one or two, and $6.6 \%$ more than six flares during two years. No associations were found between patient characteristics (e.g. age, time after initial diagnosis, crystal diagnosis, ULT use, cardiovascular co-morbidity, diuretic use) to differentiate patients with "no-or-1-flare" and "2-or-more-flares" per two years.

Conclusions: Occurrence of flares in this stringently observed primary care cohort of patients with gout was very low, even if most patients did not use prophylactic ULT. Only $6.6 \%$ of patients experienced $>6$ flares in two years. Patients, in particular those not using ULT and with a low flare frequency (the majority), may reflect individuals with a distinctive (moderate) disease activity. They probably do not fulfill the general accepted advancing course of gout with ongoing and increasing flare frequency, MSU deposition, tophus formation, and joint damage. Immediate lifelong ULT for them may not be automatically indicated. Our study shows that suboptimal gout management in the perception of rheumatologists (secondary care) does not lead to an abundant number of gout flares in primary care patients. This urges to caution when recommendations based upon secondary care guide lines are advised for primary care patients with gout.

\section{References:}

[1] Doherty M, et al. Ann Rheum Dis 2012;71:1765-70.

[2] Dalbeth N, et al. Arthritis Rheumatol 2017;69:22-34.

[3] McLean RM, et al. Ann Intern Med 2017:166:73-4.

[4] Morillon MB, et al. BMJ Open 2016;6:e012026.

Disclosure of Interest: None declared

DOI: 10.1136/annrheumdis-2017-eular.6295

\section{THU0429 CLINICAL SIGNIFICANCE OF INCREASED SERUM LEVELS OF FGF23 IN FIBROUS DYSPLASIA}

H. Florez $^{1}$, S. Mandelikova ${ }^{1}$, X. Filella ${ }^{2}$, A. Monegal ${ }^{1}$, N. Guañabens ${ }^{1}$, P. Peris ${ }^{1} .{ }^{1}$ Rheumatology Department, ${ }^{2}$ Clinical Biochemistry Department, Hospital Clinic, Barcelona, Spain

Background: Fibrous dysplasia of bone (FD) is an uncommon skeletal disorder, caused by missense mutations of the GNAS1 gene and is characterized by the development of fibro-osseous lesions that replace normal bone. FD can present with a broad spectrum of clinical manifestations, including the development of hypophosphatemic osteomalacia which is due to the production of the phosphaturic hormone fibroblast growth factor 23 (FGF-23) by the dysplastic bone tissue. Nevertheless, the prevalence of this clinical complication is not well known.

Objectives: To analyse the serum levels of FGF-23 in patients with FD and determine their relationship with the extension and activity of the disease, as well as with serum phosphate levels.

Methods: Twelve patients (7F:5M) with FD with a mean age of $50.67 \pm 16.4$ years (24-79) were included. The clinical reports of the patients were reviewed, with special attention to the extension and activity of the disease, number and location of the affected bones, clinical complications and treatments received. Serum FGF-23 values were recorded in all subjects (determined by Immunotopics, CA, USA [measuring FGF23 C- terminal], normal value $<130 \mathrm{RU} / \mathrm{ml}$ ), as well as serum phosphate and calcium values, bone turnover markers and their evolution with treatment.

Results: Serum levels of FGF-23 were increased (>130 RU/ml) in 6/12 patients $(50 \%)$. In patients with and without high FGF-23 levels the number of affected bones $(2.2 \pm 2$ vs. $1.9 \pm 1$, respectively) and the skeletal locations of FD were similar as was the age in both groups of patients $(48.2 \pm 14$ vs. $53.2 \pm 19$ years). In addition, FD disease activity and extension were similar in the two groups as were the bone turnover marker values (FAO, PINP and CTx). Strikingly, differences between serum phosphate values were not observed between the two groups (FGF23 > 130: $3.9 \pm 0.9 \mathrm{mg} / \mathrm{dl}$ vs. FGF23 <130: $3.5 \pm 0.6 \mathrm{mg} / \mathrm{dl}$ ). Indeed, none of the patients with high FGF-23 levels had low serum phosphate values. Following bisphosphonate (zoledronate) treatment, there were no significant changes in FGF-23 values. Nevertheless, an increase of $123 \%$ was found in one patient receiving denosumab, although hypophosphatemia was not associated.

Conclusions: Patients with FD frequently present elevated FGF-23 values with no effects on serum phosphate levels, thereby suggesting the presence of an alteration in processing this protein in the dysplastic bone tissue in this disease. The role of denosumab treatment in FD and its repercussion on FGF-23 levels need further study.

Disclosure of Interest: None declared

DOI: 10.1136/annrheumdis-2017-eular.5261 